MATHEMATICS OF COMPUTATION

Volume 72, Number 244, Pages 2099-2110

S 0025-5718(03)01465-0

Article electronically published on May 1, 2003

\title{
A COMPUTATIONAL APPROACH FOR SOLVING
}

$$
y^{2}=1^{k}+2^{k}+\cdots+x^{k}
$$

\author{
M. J. JACOBSON, JR., Á. PINTÉR, AND P. G. WALSH
}

\begin{abstract}
We present a computational approach for finding all integral solutions of the equation $y^{2}=1^{k}+2^{k}+\cdots+x^{k}$ for even values of $k$. By reducing this problem to that of finding integral solutions of a certain class of quartic equations closely related to the Pell equations, we are able to apply the powerful computational machinery related to quadratic number fields. Using our approach, we determine all integral solutions for $2 \leq k \leq 70$ assuming the Generalized Riemann Hypothesis, and for $2 \leq k \leq 58$ unconditionally.
\end{abstract}

\section{INTRODUCTION}

É. Lucas [18] proved that the diophantine equation

$$
y^{2}=1^{2}+2^{2}+\cdots+x^{2}
$$

has only the solutions $x=y=1$ and $x=24, y=70$. Schäffer [20] furthered this work by studying the more general equation

$$
y^{q}=1^{k}+2^{k}+\cdots+x^{k} .
$$

The main result of this work was a proof that the only positive integers $(k \geq 1$, $q>1$ ) for which this equation has infinitely many solutions are

$$
(k, q) \in\{(1,2),(3,2),(3,4),(5,2)\} .
$$

Schäffer also made the following

Conjecture $1.1([20])$. Let $k$ and $q>1$ be positive integers, with $(k, q)$ not in the above list. Then apart from the solution $(x, y)=(24,70)$ when $k=q=2$, the only solution to equation (2) is the trivial solution $x=y=1$.

In recent years there have been numerous papers on this topic (see [2], [3], 7], 10], 22 ] ). The interested reader may wish to refer to the notes at the end of chapter 10 in 21 .

In a recent paper [4, the authors prove the following

Received by the editor June 5, 2001.

2000 Mathematics Subject Classification. Primary 11D25, 11J86.

Key words and phrases. Diophantine equations, elliptic curves, quadratic fields.

The first and third authors are supported by the Natural Sciences and Engineering Research Council of Canada.

The second author is supported by the Hungarian National Foundation for Scientific Research, grants T29330, F34891, and FKFP-066-2001. 
Theorem 1.1 (4]). For $k \geq 2$ even, the equation

$$
y^{2}=1^{k}+2^{k}+\cdots+(x-1)^{k}
$$

has at most $\max \left\{c_{1}, 9^{k}\right\}$ solutions in integers $x$ and $y$, where $c_{1}$ is an effectively computable absolute constant.

Although this theorem does not prove Conjecture 1.1, its proof provides a methodology for finding all integer solutions to equation (3). In particular, this was the goal in [19], wherein Pintér found all solutions to (3) for $k \in\{2,4,6,8,10,14\}$.

The purpose of the present paper is to improve on the result in [19] by reducing the problem to finding all integer points on a certain class of quartic equations, and appealing to the ideas from [1] to improve the computation. As a result of this reduction, we can improve upon [19] as follows.

Theorem 1.2. For $2 \leq k \leq 58$ and $k$ even, the only positive integer solution $(x, y)$ to equation (3) is the trivial solution $(x, y)=(2,1)$, except in the case $k=2$, for which there is the anomalous solution $(x, y)=(25,70)$. Under the assumption of the Generalized Riemann Hypothesis (GRH), the result also holds in the range $60 \leq k \leq 70$.

The dependence of Theorem 1.2 on the GRH is due to our algorithm's use of a conditional subexponential algorithm for computing the regulator of a real quadratic field [11. This is explained in more detail on Section 5

\section{Reduction to a FAMily of QUARTiC Diophantine EQUations}

We begin by following the argument in [19]. For $k>0$, we define $S_{k}(x)$ to be the polynomial

$$
S_{k}(x)=1^{k}+2^{k}+\cdots+(x-1)^{k} .
$$

It is well known that for $k$ even, $(k+1) S_{k}(x)=B_{k+1}(x)$, where $B_{k+1}(x)$ represents the $k+1$-st Bernoulli polynomial (see [8] for details on Bernoulli numbers and polynomials). Suppose that $k \geq 2$ is even, and that $(x, y)$ is a positive integer solution to $y^{2}=S_{k}(x)$. From the above remark we have

$$
\begin{aligned}
(k+1) y^{2} & =(k+1) S_{k}(x) \\
& =B_{k+1}(x)=\left(\begin{array}{c}
k+1 \\
1
\end{array}\right) B_{k} x+\left(\begin{array}{c}
k+1 \\
3
\end{array}\right) B_{k-2} x^{3}+\cdots+x^{k+1},
\end{aligned}
$$

where $B_{i}(i \geq 0)$ is the $i$ th Bernoulli number. Define $d_{k}$ to be the minimal positive integer such that $d_{k}(k+1) S_{k}(x)$ has integer coefficients. It follows that

$$
d_{k}(k+1) y^{2}=x\left((k+1) d_{k} B_{k}+x^{2} f(x)\right),
$$

with $f(X)$ a polynomial with integer coefficients. Therefore, $x=a u^{2}$ with $u \geq 1$, $a \geq 1$ square-free, and $a$ divides $(k+1) d_{k}^{2} B_{k}$.

From the relation

$$
B_{k+1}(X)=(-1)^{k+1} B_{k+1}(1-X),
$$

we deduce that $x-1=c w^{2}$, where $w \geq 1, c \geq 1$ is square-free, and $c$ also divides $(k+1) d_{k}^{2} B_{k}$. For future reference, we will define $A_{k}$ as

$$
A_{k}=\left\{p \text { prime }|p|(k+1) d_{k}^{2} B_{k}\right\} .
$$

We now wish to deduce a similar result for the integer $2 x-1$. We will use the following two properties of Bernoulli polynomials $(i \geq 1)$ : 
1. $B_{i+1}^{\prime}(X)=(i+1) B_{i}(X)$;

2. $B_{i}(1 / 2)=\left(2^{1-i}-1\right) B_{i}$.

By Taylor's formula

$$
B_{k+1}(X)=\sum_{i=0}^{k+1} \frac{B_{k+1}^{(i)}(1 / 2)}{i !}(X-1 / 2)^{i},
$$

and so, by applying the above properties, we obtain

$$
B_{k+1}(X)=\sum_{i=1}^{k+1}\left(\begin{array}{c}
k+1 \\
i
\end{array}\right)\left(2^{i-k}-1\right) B_{k+1-i} \cdot 2^{-i}(2 X-1)^{i} .
$$

Define $e_{k}$ to be the smallest positive integer such that

$$
e_{i} \sum_{i=1}^{k+1}\left(\begin{array}{c}
k+1 \\
i
\end{array}\right)\left(2^{i-k}-1\right) B_{k+1-i} \cdot 2^{-i}(2 X-1)^{i}
$$

is a polynomial with integer coefficients in the variable $(2 X-1)$. Therefore, as above, we obtain

$$
e_{k}(k+1) y^{2}=(2 x-1)\left(e_{k}(k+1)\left(2^{1-k}-1\right) B_{k}+(2 x-1) g(x)\right),
$$

where $g(X)$ is a polynomial with integer coefficients. Therefore, $2 x-1=b v^{2}$, where $v \geq 1, b \geq 1$ is square-free, and $b$ is a product of primes which are divisors of the integers

$$
\left\{e_{k}, k+1,2^{k-1}-1 \text {, numerator }\left(B_{k}\right)\right\} .
$$

For future reference, we will define the set of primes $C_{k}$ to be those odd primes which divide any one of the integers in (4).

Let $Z=2 x-1, \omega=a b c$, and $W=2 u v w$, then $(Z, W)$ is an integer point on the elliptic curve

$$
Z^{3}-Z=\omega W^{2}
$$

Thus, the problem now reduces to finding all integer points on all possible curves of the form (5), where $\omega$ ranges over the square-free integers composed of primes in $A_{k} \cup C_{k}$. It is well known that this can be accomplished in several ways, the most efficient likely being the implementation of Gebel, Pethö, and Zimmer 9. The problem here is that the size of the sets $A_{k}$ and $C_{k}$ and the size of their elements grow very quickly with $k$, thereby requiring not only the diophantine resolution of many elliptic curves but, more importantly, curves whose defining parameters are indeed quite large.

The main point in the present paper is to provide an alternative approach. With $a, b, c, u, v, w$ as defined above, let $d=a c$ and $z=2 u w$. Then $(X, Y)=(v, z)$ is a point on the quartic curve

$$
b^{2} X^{4}-d Y^{2}=1
$$

\section{The Diophantine equation $b^{2} X^{4}-d Y^{2}-1$}

Equation (6) has been studied in considerable detail. We state two theorems on its solubility which will provide the basis for our algorithm to solve equation (3). The first theorem can be found in [6], while the second can be found in [1]. 
Lemma 3.1 ([6]). Let $d$ denote a square-free positive integer, and let $T+U \sqrt{d}$ denote the minimal solution to the equation $X^{2}-d Y^{2}=1$. For $k \geq 1$, let $T_{k}+$ $U_{k} \sqrt{d}=(T+U \sqrt{d})^{k}$. If $(X, Y)=(v, z)$ is a solution to $X^{4}-d Y^{2}=1$, then $v^{2}=T_{1}$ or $v^{2}=T_{2} . T_{1}$ and $T_{2}$ are both squares only for $d=1785$.

Lemma $3.2([1])$. Let $b>1$ and $d>1$ be square-free integers, then the equation $b^{2} X^{4}-d Y^{2}=1$ has at most one solution in positive integers $(X, Y)$. If a solution exists, then $b X^{2}=T_{\beta(b)}$, where $\beta(b)$ is defined to be the minimal index $k$ for which $b$ divides $T_{k}$ (note that $\beta(b)$ may or may not exist).

\section{The ALGORITHM}

In this section we describe an algorithm based on the results of the previous section for finding all integer points $(X, Y)$ on those curves $b^{2} X^{4}-d Y^{2}=1$, as $b$ and $d$ range over the set of square-free integers composed of primes in $C_{k}$ and $A_{k}$, respectively. The bottleneck in the algorithm is easily seen to be the computation of the minimal solution to the Pell equation $X^{2}-d Y^{2}=1$. Once this has been computed for a given value $d$, the remaining computations are completed with relative ease. We remark that the steps involved in the algorithm are in some ways dictated by properties of solutions to Pell equations, and so the interested reader may wish to consult [15] or [23] for further details.

Algorithm 4.1. For each square-free integer $d>1$ composed of primes in $A_{k}$, perform the following steps:

1. Compute $T+U \sqrt{d}$, the minimal solution to $X^{2}-d Y^{2}=1$.

2. For each $p \in C_{k}$ such that $p \nmid d$, determine if $(\beta(p)$ exists, and if so, then compute $\beta(p)$.

3. Let $\left\{p_{1}, \ldots, p_{t}\right\}$ denote those primes $p$ for which $\beta(p)$ exists. Partition the set $\left\{p_{1}, \ldots, p_{t}\right\}$ into equivalence classes, where $p_{i}$ and $p_{j}$ are in the same class if and only if $\beta\left(p_{i}\right)$ and $\beta\left(p_{j}\right)$ are exactly divisible by the same power of 2 .

4. This step is repeated for each equivalence class determined in Step 3. Let $C$ denote an equivalence class. For each square-free integer $m>1$, divisible only by primes in $C$, define $\beta(m)=\operatorname{lcm}_{p \mid m}\{\beta(p)\}$. (Note that $m$ divides some $T_{i}$ if and only if the prime divisors of $m$ are all in the same equivalence class).

5. Determine if $T_{1}$ or $T_{2}$ is a square. For each $m>1$ divisible only by primes in $C_{k}$ such that $\beta(m)$ exists, determine if $\left(T_{\beta(m)}\right) / m$ is a square.

4.1. Remarks concerning the implementation of Algorithm 4.1. The main difficulty in implementing Algorithm 4.1 is computing and working with $\eta=T+$ $U \sqrt{d}$, the minimal solution of $X^{2}-d Y^{2}=1$. In general, $\log \eta$ is $O(O \sqrt{d})$, so except for small values of $d$ it is usually infeasible to compute $T$ and $U$ explicitly. However, given a sufficiently accurate approximation of $\log \eta$, and explicit representation of $\eta$ called a compact representation can be computed in polynomial time [5]. This compact representation has the form

$$
\eta=\gamma \prod_{j=1}^{k}\left(\alpha_{j} / d_{j}\right)^{2^{k-j}}
$$


where $d_{j} \in \mathbb{Z}^{+}, \gamma \in \mathcal{O}_{d}, \alpha_{j}=\left(a_{j}+b_{j} \sqrt{\Delta}\right) / 2 \in \mathcal{O}_{d}, a_{j}, b_{j} \in \mathbb{Z}(j=1,2, \ldots, k), \Delta$ is the discriminant of the quadratic field $\mathbb{Q}(\sqrt{d})$, and $\mathcal{O}_{d}$ is the ring of integers of $\mathbb{Q}(\sqrt{d})$. For our purposes, having a compact representation of $\eta$ is almost always sufficient. Indeed, unless a solution of $b^{2} X^{4}-d Y^{2}=1$ is actually found, we only need to know $T$ and $U$ modulo various primes, a computation which can be carried out very efficiently using the methods in [14] once a compact representation has been obtained.

To compute $\log \eta$, we first compute an approximation of $R=\log \varepsilon_{d}$, the regulator of $\mathbb{Q}(\sqrt{d})$. Since $\eta=\varepsilon_{d}^{\nu}$ with $\nu \in\{1,2,3,6\}$ (see, for example, [13]) we can easily compute a compact representation of $\eta$ from $\nu$ and $R$. Computing the regulator $R$ is in fact the most time-consuming part of the algorithm. The best unconditional algorithm has complexity $O\left(d^{1 / 5+\varepsilon}\right)$ [16], [12, but for values of $d>10^{20}$, this is much too inefficient. For these values of $d$, we used the subexponential algorithm described in [11.

Once a compact representation of $\eta$ has been computed, the next step is to compute $\beta(p)$ for each prime $p \in C_{k}$ not dividing $d$. If $\beta(p)$ exists, it must divide $(p-(d / p)) / 4$ (see [23]), so we only have to compute $T_{l} \bmod p$ for each $l \mid(p-(d / p)) / 4$ and take $\beta(p)$ to be the smallest such $l$ for which $T_{l} \equiv 0(\bmod p)$. Given the compact representation of $\eta$, we can use the methods of [14] to compute $T \bmod p$. Using the identities $T_{2 n}=T_{n}^{2}+Y_{n}^{2} d$ and $U_{2 n}=2 T_{n} U_{n}$ (see, for example, [23]), $T_{l}$ can be computed efficiently using an analogue of the well-known binary exponentiation method given the binary representation of $l$. Computing the set of admissible values of $m$ and $\beta(m)$ (Step 3 and 4 ) is straightforward after the $\beta(p)$ have been computed.

The last step of the algorithm is to determine whether $T_{1}, T_{2}$, or $\left(T_{\beta(m)}\right) / m$ is a square. Each square value yields an integral solution of the elliptic curve (5) where $\omega=m d, Z=T_{1}, T_{2}$ or $T_{\beta(m)}$, and $W=\sqrt{\left(Z^{3}-Z\right) / w}$, which can in turn be tested as to whether a solution of (3) has been found. In general, computing $T_{l}$ explicitly is infeasible, but once again we use compact representations to solve this problem. In the vast majority of cases, especially for large values of $d$, we expect that $T_{l}$ will not be a square. Thus, we expect that computing the Legendre symbol $\left(T_{l} / p\right)$ for a number of small primes $p$ will eventually yield a value of -1 , demonstrating that $T_{l}$ is not square. For this test, we only need $T_{l} \bmod p$ for each prime $p$; again, the methods described in [14] can be used for this purpose. If we have tried 30 primes $p$ and we get $\left(T_{l} / p\right)=1$ for each of them, then we assume that $T_{l}$ is in fact square and are forced to compute it explicitly, which was in practice a rare occurrence (only occuring for small values of $T_{l}$ ).

\section{Computational Results}

We have implemented Algorithm 4.1 from the previous section and used it to numerically verify Conjecture 1.1 for $2 \leq k \leq 70$. Our code is written with the $\mathrm{C}++$ library LiDIA [17] and compiled with the GNU g++ complier version 2.91.66. The program was run on an $800 \mathrm{MHz}$ Pentium processor running Linux. The entire computation took just over 11 days. Complete listings of the sets $A_{k}$ and $C_{k}$, and all the integral points found on the curve (5) can be found in Table 2 and Table 3 in Appendix A. For each point in Table 3, we list the values of $k$ for which that point was found. The only solutions of (3) we found were the trivial solution for each value of $k$, and for $k=2$ the anomalous solution $x=24, y=70$. 
Because the correctness of the subexponential algorithm described in [11] for computing $R$ is conditional on the Generalized Riemann Hypothesis, the correctness of our results is also conditional for those values of $k$ which made use of this algorithm. In practice, the subexponential algorithm does return a multiple of the regulator, but in order for the values of $\beta(p)$ to be correct, it is necessary that the value returned is the actual regulator so that $\eta$ is the fundamental solution of Pell's equation.

In our implementation, we used the subexponential algorithm to compute $R$ whenever $d>10^{20}$ and the unconditional baby step-giant step variant of complexity $O\left(d^{1 / 5+\varepsilon}\right)$ [16], [12] otherwise. For $k \leq 34$ there were no values of $d>10^{20}$, so these results are unconditionally correct. For $36 \leq k \leq 58$, we were forced to use the subexponential algorithm to compute some of the regulators, but we were nevertheless able to verify unconditionally that these regulators were correct. Given a multiple $S$ of the regulator produced by the subexponential algorithm, we first verify that $R>S^{2 / 3}$ using a baby step-giant step algorithm. Then, for each prime $p$ such that $S / p>S^{2 / 3}$, we verify that $S / p$ is not also a multiple of $R$ by checking that the ideal closest to $S / p$ from $\mathcal{O}_{d}$ is not $\mathcal{O}_{d}$. Both stages of the algorithm take time $O\left(S^{1 / 3}\right)$ and, assuming that $S=R$, we get an overall run time of $O\left(d^{1 / 6+\varepsilon}\right)$ for verifying that the value produced by the subexponential algorithm is indeed the regulator. This method for unconditionally computing $r$ is the subject of ongoing research, and will be described in more detail in a forthcoming paper.

In Table1 we present various run time statistics from the computation. For each even value of $k$ between 2 and 70, we list the time to execute Algorithm 4.1 (Time), the maximum decimal length of $d$ during the course of the algorithm $(\max d)$, the number of values of $d$ generated (\#d=2 $2^{\left|A_{k}\right|}-1$ ), the number of $d$ values for which the assumption of the GRH is necessary (\# GRH), and the time to verify that the value of $R$ produced is unconditionally correct for all $d$ (Verify Time). For the times, seconds, minutes, hours, and days are denoted by "s", "m", "h", and "d", respectively.

Clearly, two factors prevent us from extending this table much further. First of all, the number of $d$ values which must be processed by Algorithm 4.1 is exponential in $k$. Even for $k \leq 70$ we have three cases where 2047 values of $d$ were generated. The regulator has to be evaluated for each of them, and while a few examples of the required sizes can be computed efficiently, evaluating 1024 regulators for $d$ having between 20 and 51 decimal digits, for example, is very time-consuming.

Secondly, the $d$ values themselves get larger as $k$ increases. Indeed, to do $k=72$ would require processing 2047 values of $d$, the largest having 64 decimal digits. Computing the regulator for a single $d$ value of this size takes over 2 hours, so the amount of computation required to process all 2047 values of $d$ for $k=72$ would be significantly greater than for the previous values of $k$.

As mentioned earlier, we were successful in removing the dependence on the GRH from our results for $k \leq 58$. Although we spent as much as 3.75 days verifying our results for a single $k$ value, even this would not have been possible without the new $O\left(d^{1 / 6+\varepsilon}\right)$ method mentioned above. Extending this part of the computation would also be difficult. For $k \leq 58$, we had to compute regulators unconditionally for $d$ values with as many as 39 decimal digits. To do the same for $k=60$ would require handling $d$ values with up to 51 decimal digits, a computation which currently appears to be out of reach with the available methods. 
TABLE 1. Run time statistics.

\begin{tabular}{|r|r|r|r|r|r|}
\hline$k$ & Time & $\max d$ & $\# d$ & \# GRH & Verify Time \\
\hline \hline 2 & $0.01 \mathrm{~s}$ & 1 & 3 & & \\
4 & $0.03 \mathrm{~s}$ & 2 & 7 & & \\
6 & $0.09 \mathrm{~s}$ & 2 & 7 & & \\
8 & $0.07 \mathrm{~s}$ & 2 & 7 & & \\
10 & $0.26 \mathrm{~s}$ & 3 & 15 & & \\
12 & $1.66 \mathrm{~s}$ & 7 & 63 & & \\
14 & $0.25 \mathrm{~s}$ & 3 & 15 & & \\
16 & $0.91 \mathrm{~s}$ & 7 & 31 & & \\
18 & $2.08 \mathrm{~s}$ & 9 & 63 & & \\
20 & $3.46 \mathrm{~s}$ & 9 & 127 & & \\
22 & $5.92 \mathrm{~s}$ & 9 & 127 & & \\
24 & $16.55 \mathrm{~s}$ & 12 & 127 & & \\
26 & $2.23 \mathrm{~s}$ & 9 & 31 & & \\
28 & $20.53 \mathrm{~s}$ & 14 & 127 & & \\
30 & $2 \mathrm{~m} 38.32 \mathrm{~s}$ & 18 & 255 & & \\
32 & $4 \mathrm{~m} 40.55 \mathrm{~s}$ & 18 & 511 & & \\
34 & $27.32 \mathrm{~s}$ & 15 & 63 & & \\
36 & $6 \mathrm{~m} 41.59 \mathrm{~s}$ & 27 & 255 & 127 & \\
38 & $4 \mathrm{~m} 58.13 \mathrm{~s}$ & 19 & 127 & & \\
40 & $5 \mathrm{~m} 50.06 \mathrm{~s}$ & 26 & 255 & 64 & \\
42 & $3 \mathrm{~m} 35.36 \mathrm{~s}$ & 27 & 127 & 64 & \\
44 & $39 \mathrm{~m} 27.89 \mathrm{~s}$ & 27 & 1023 & 173 & $37 \mathrm{~m} 42.75 \mathrm{~s}$ \\
46 & $9 \mathrm{~m} 38.90 \mathrm{~s}$ & 29 & 255 & 64 & $57 \mathrm{~m} 27.35 \mathrm{~s}$ \\
48 & $18 \mathrm{~m} 46.91 \mathrm{~s}$ & 34 & 511 & 256 & $8 \mathrm{~h} 49 \mathrm{~m} 34.44 \mathrm{~s}$ \\
50 & $14 \mathrm{~m} 44.81 \mathrm{~s}$ & 31 & 255 & 86 & $2 \mathrm{~h} 39 \mathrm{~m} 44.29 \mathrm{~s}$ \\
52 & $57 \mathrm{~m} 56.25 \mathrm{~s}$ & 35 & 1023 & 391 & $12 \mathrm{~h} 4 \mathrm{~m} 5.38 \mathrm{~s}$ \\
54 & $29 \mathrm{~m} 12.05 \mathrm{~s}$ & 37 & 511 & 241 & $1 \mathrm{~d} 6 \mathrm{~h} 4 \mathrm{~m} 25.78 \mathrm{~s}$ \\
56 & $31 \mathrm{~m} 49.05 \mathrm{~s}$ & 39 & 511 & 256 & $3 \mathrm{~d} 17 \mathrm{~h} 5 \mathrm{~m} 14.47 \mathrm{~s}$ \\
58 & $38 \mathrm{~m} 49.77 \mathrm{~s}$ & 39 & 511 & 243 & $2 \mathrm{~d} 18 \mathrm{~h} 2 \mathrm{~m} 6.00 \mathrm{~s}$ \\
60 & $13 \mathrm{~h} 13 \mathrm{~m} 16.35 \mathrm{~s}$ & 51 & 2047 & 1024 & \\
62 & $2 \mathrm{~h} 57 \mathrm{~m} 20.11 \mathrm{~s}$ & 43 & 2047 & 1074 & \\
64 & $1 \mathrm{~h} 9 \mathrm{~m} 54.65 \mathrm{~s}$ & 47 & 255 & 125 & \\
66 & $19 \mathrm{~h} 34 \mathrm{~m} 6.74 \mathrm{~s}$ & 53 & 1023 & 512 & \\
68 & $7 \mathrm{~h} 8 \mathrm{~m} 6.09 \mathrm{~s}$ & 49 & 2047 & 1101 & \\
70 & $6 \mathrm{~h} 42 \mathrm{~m} 43.14 \mathrm{~s}$ & 53 & 511 & 383 & \\
\hline total & $2 \mathrm{~d} 7 \mathrm{~h} 15 \mathrm{~m} 38.08 \mathrm{~s}$ & 53 & 14873 & 6187 & $81 \mathrm{~d} 19 \mathrm{~h} 56 \mathrm{~m} 50.43 \mathrm{~s}$ \\
\hline & & & & & \\
\hline
\end{tabular}

\section{A. Appendix}

Table 2 contains the complete sets $A_{k}$ and $C_{k}$ described in Section [2] Table 3 contains all the integral points found on the elliptic curve (5) during the course of our computations. For each point in Table 3, we list the values of $k$ for which that point was found. 
TABle $2 . A_{k}$ and $C_{k}$ values.

\begin{tabular}{|r|l|}
\hline$k$ & \\
\hline \hline 2 & $A_{k}=\{2,3\}, C_{k}=\{3\}$ and $C_{k}$ \\
4 & $A_{k}=\{2,3,5\}, C_{k}=\{3,5,7\}$ \\
6 & $A_{k}=\{2,3,7\}, C_{k}=\{3,7,31\}$ \\
8 & $A_{k}=\{2,3,5\}, C_{k}=\{3,5,127\}$ \\
10 & $A_{k}=\{2,3,5,11\}, C_{k}=\{3,5,7,11,73\}$ \\
12 & $A_{k}=\{2,3,5,7,13,691\}, C_{k}=\{3,5,7,13,23,89,691\}$ \\
14 & $A_{k}=\{2,3,5,7\}, C_{k}=\{3,5,7,8191\}$ \\
16 & $A_{k}=\{2,3,5,17,3617\}, C_{k}=\{3,5,7,17,31,151,3617\}$ \\
18 & $A_{k}=\{2,3,5,7,19,43867\}, C_{k}=\{3,5,7,19,43867,131071\}$ \\
20 & $A_{k}=\{2,3,5,7,11,283,617\}, C_{k}=\{3,5,7,11,283,617,524287\}$ \\
22 & $\left.A_{k}=\{2,3,5,11,23,131,593\}, C_{k}=3,5,7,11,23,127,131,337,593\right\}$ \\
24 & $A_{k}=\{2,3,5,7,13,103,2294797\}$, \\
& $C_{k}=\{3,5,7,13,47,103,178481,2294797\}$ \\
26 & $\left.A_{k}=2,3,7,13,657931\right\}, C_{k}=\{3,7,13,31,601,1801,657931\}$ \\
28 & $A_{k}=\{2,3,5,7,29,9349,362903\}$, \\
& $C_{k}=\{3,5,7,29,73,9349,262657,362093\}$ \\
30 & $A_{k}=\{2,3,5,7,11,31,1721,1001259881\}$, \\
& $C_{k}=\{3,5,7,11,31,233,1103,1721,2089,1001259881\}$ \\
32 & $A_{k}=\{2,3,5,7,11,17,37,683,305065927\}$, \\
& $C_{k}=\{3,5,7,11,17,37,683,305065927,2147483647\}$ \\
34 & $A_{k}=\{2,3,5,7,17,151628697551\}$, \\
& $C_{k}=\{3,5,7,17,23,89,599479,151628697551\}$, \\
36 & $A_{k}=\{2,3,5,7,13,19,37,26315271553053477373\}$, \\
& $C_{k}=\{3,5,7,13,19,31,37,71,127,122921,26315271553053477373\}$ \\
38 & $A_{k}=\{2,3,5,7,13,19,154210205991661\}$, \\
40 & $C_{k}=\{3,5,7,13,19,223,616318177,154210205991661\}$ \\
40 & $A_{k}=\{2,3,5,7,11,41,137616929,1897170067619\}$, \\
42 & $C_{k}=\{3,5,7,11,41,79,8191,121369,137616929,1897170067619\}$ \\
& $A_{k}=\{2,3,5,7,11,43,1520097643918070802691\}$, \\
& $C_{k}=\{3,5,7,11,43,13367,164511353,1520097643918070802691\}$ \\
& $A_{k}=\{2,3,5,7,11,23,59,8089,2947939,1798482437\}$, \\
& $C_{k}=\{3,5,7,11,23,59,431,8089,9719,2099863,2947939,1798482437\}$ \\
& $A_{k}=\{2,3,5,7,23,47,383799511,67568238839737\}$, \\
& $C_{k}=\{3,5,7,23,31,47,73,151,631,23311,383799511,67568238839737\}$ \\
& $A_{k}=\{2,3,5,7,13,17,653,56039,153289748932447906241\}$, \\
& $C_{k}=\{3,5,7,13,17,653,2351,4513,56039,13264529$, \\
& $A_{k}=\{2,3,5,11,13,17,417202699,47464429777438199\}$, \\
$A_{k}=\{3,5,11,13,17,127,417202699,4432676798593,47464429777438199\}$ \\
$A_{k}=\{3,5,5,11,13,53,577,58741,401029177,4534045619429\}$, \\
\end{tabular}


A COMPUtATional APPROACH FOR SOLVING $y^{2}=1^{k}+2^{k}+\cdots+x^{k} \quad 2107$

TABle 2. (Continued)

\begin{tabular}{|c|c|}
\hline$k$ & $A_{k}$ and $C_{k}$ \\
\hline 56 & $\begin{aligned} A_{k}=\{2,3,5,7,19,29,113161,163979,19088082706840550550313\} \\
C_{k}=\{3,5,7,19,23,29,31,89,881,3191,113161,163979,201961 \\
19088082706840550550313\}\end{aligned}$ \\
\hline 58 & $\begin{aligned} A_{k}=\{2,3,5,29,59,67,186707,6235242049,37349583369104129\} \\
C_{k}=\{3,5,7,29,59,67,32377,186707,524287,1212847,6235242049 \\
37349583369104129\}\end{aligned}$ \\
\hline 60 & $\begin{aligned} & A_{k}=\{2,3,5,7,11,13,31,61,2003,5549927 \\
&109317926249509865753025015237911\} \\
& C_{k}=\{3,5,7,11,13,31,61,2003,179951,5549927,3203431780337 \\
&109317926249509865753025015237911\}\end{aligned}$ \\
\hline 62 & $\begin{array}{c}A_{k}=\{2,3,5,7,11,13,31,157,266689,329447317,28765594733083851481\} \\
C_{k}=\{3,5,7,11,13,31,157,266689,329447317,28765594733083851481 \\
28765594733083851481\}\end{array}$ \\
\hline 64 & $\begin{array}{c}A_{k}=\{2,3,5,11,13,17,1226592271,87057315354522179184989699791727\} \\
C_{k}=\{3,5,7,11,13,17,73,127,337,92737,649657,1226592271, \\
87057315354522179184989699791727\}\end{array}$ \\
\hline 66 & $\begin{array}{c}A_{k}=\{2,3,5,7,11,17,23,67,839 \\
159562251828620181390358590156239282938769\} \\
C_{k}=\{3,5,7,11,17,23,31,67,839,8191,145295143558111 \\
159562251828620181390358590156239282938769\}\end{array}$ \\
\hline 68 & $\begin{array}{c}A_{k}=\{2,3,5,7,17,23,37,101,123143,1822329343, \\
5525473366510930028227481\}, \\
C_{k}=\{3,5,7,17,23,37,101,123143,193707721,1822329343,761838257287, \\
5525473366510930028227481\}\end{array}$ \\
\hline 70 & $\begin{array}{c}A_{k}=\{2,3,5,7,11,71,688531,20210499584198062453 \\
3090850068576441179447\} \\
C_{k}=\{3,5,7,11,47,71,178481,688531,10052678938039 \\
20210499584198062453,3090850068576441179447\}\end{array}$ \\
\hline
\end{tabular}

Table 3. Points on $Z^{3}-Z=\omega W^{2}$.

\begin{tabular}{|r||r|r|r|}
\hline & $\omega$ & \multicolumn{1}{|c|}{$Z$} & $W$ \\
\hline \hline $4,8-24,28-70$ & 5 & 9 & 12 \\
$2-70$ & 6 & 3 & 2 \\
$2-70$ & 6 & 49 & 140 \\
$4,8-24,28-70$ & 15 & 4 & 2 \\
$4,6,10-48,52-70$ & 21 & 7 & 4 \\
$28,56,58$ & 22 & 99 & 210 \\
$4,8-24,28-70$ & 29 & 9801 & 180180 \\
$10,20,22,30,32,40-44,50-54,60-66,70$ & 5 & 2 \\
$16,32,34,48,50,64,66,68$ & 34 & 17 & 12 \\
$12,24,26,36,38,48,50,52,60-64$ & 39 & 25 & 20 \\
46 & 141 & 48 & 28 \\
$28,56,58$ & 145 & 289 & 408 \\
$18,36,38,54,56$ & 190 & 19 & 6 \\
\hline
\end{tabular}


TABle 3] (Continued)

\begin{tabular}{|c|c|c|c|}
\hline$k$ & $\omega$ & $Z$ & $W$ \\
\hline 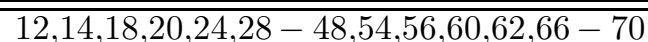 & $\overline{210}$ & $\overline{15}$ & 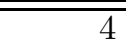 \\
\hline $12,14,18,20,24,28-48,54,56,60-70$ & 210 & 1681 & 4756 \\
\hline $16,32,34,48,50,64-68$ & 255 & 16 & 4 \\
\hline $10,20,22,30,32,40-44,50-54,60-66,70$ & 330 & 11 & 2 \\
\hline 60 & 366 & 243 & 198 \\
\hline 40 & 410 & 81 & 36 \\
\hline $50,52,60-64$ & 429 & 12 & 2 \\
\hline $30,60,62$ & 434 & 63 & 24 \\
\hline $12,24,36,38,48,60,62$ & 455 & 64 & 24 \\
\hline $16,30,36,46,56,60,62,66$ & 465 & 31 & 8 \\
\hline $12,24,26,36,38,48,60,62$ & 546 & 13 & 2 \\
\hline $12,24,26,36,38,48,60,62$ & 546 & 27 & 6 \\
\hline $32,50,64,66$ & 561 & 33 & 8 \\
\hline $28,56,58$ & 609 & 28 & 6 \\
\hline $22,44,66$ & 759 & 23 & 4 \\
\hline $12,22,34,44,56,56,66,68$ & 805 & 161 & 72 \\
\hline 36 & 889 & 127 & 48 \\
\hline 60 & 915 & 121 & 44 \\
\hline 60 & 915 & 244 & 126 \\
\hline 52 & 1154 & 577 & 408 \\
\hline $20,30,32,40-44,54,60,62,66,70$ & 1155 & 55 & 12 \\
\hline $16,32,34,48,64-68$ & 1190 & 35 & 6 \\
\hline $32,36,68$ & 1295 & 36 & 6 \\
\hline $48,50,64$ & 1326 & 51 & 10 \\
\hline 36 & 1406 & 37 & 6 \\
\hline $32,34,48,66,68$ & 1785 & 169 & 52 \\
\hline $32,34,48,66,68$ & 1785 & 57121 & 323128 \\
\hline $18,36,38,54,56$ & 1995 & 20 & 2 \\
\hline $30,60,62$ & 2170 & 125 & 30 \\
\hline $10,20,22,30,32,40-44,52,54,60-66,70$ & 2310 & 21 & 2 \\
\hline 42 & 2365 & 44 & 6 \\
\hline 36,70 & 2485 & 71 & 12 \\
\hline $22,44,66$ & 2530 & 45 & 6 \\
\hline 60,62 & 3003 & 351 & 120 \\
\hline 24 & 3090 & 59535 & 261324 \\
\hline 36,38 & 3705 & 39 & 4 \\
\hline 52 & 4134 & 53 & 6 \\
\hline 36 & 4218 & 75 & 10 \\
\hline $50,52,60-64$ & 4290 & 65 & 8 \\
\hline 40 & 4305 & 41 & 4 \\
\hline 54 & 4389 & 76 & 10 \\
\hline 28,56 & 6090 & 29 & 2 \\
\hline 46 & 6486 & 47 & 4 \\
\hline 24,52 & 7210 & 721 & 228 \\
\hline 36 & 7215 & 1444 & 646 \\
\hline
\end{tabular}


TABle 3 (Continued)

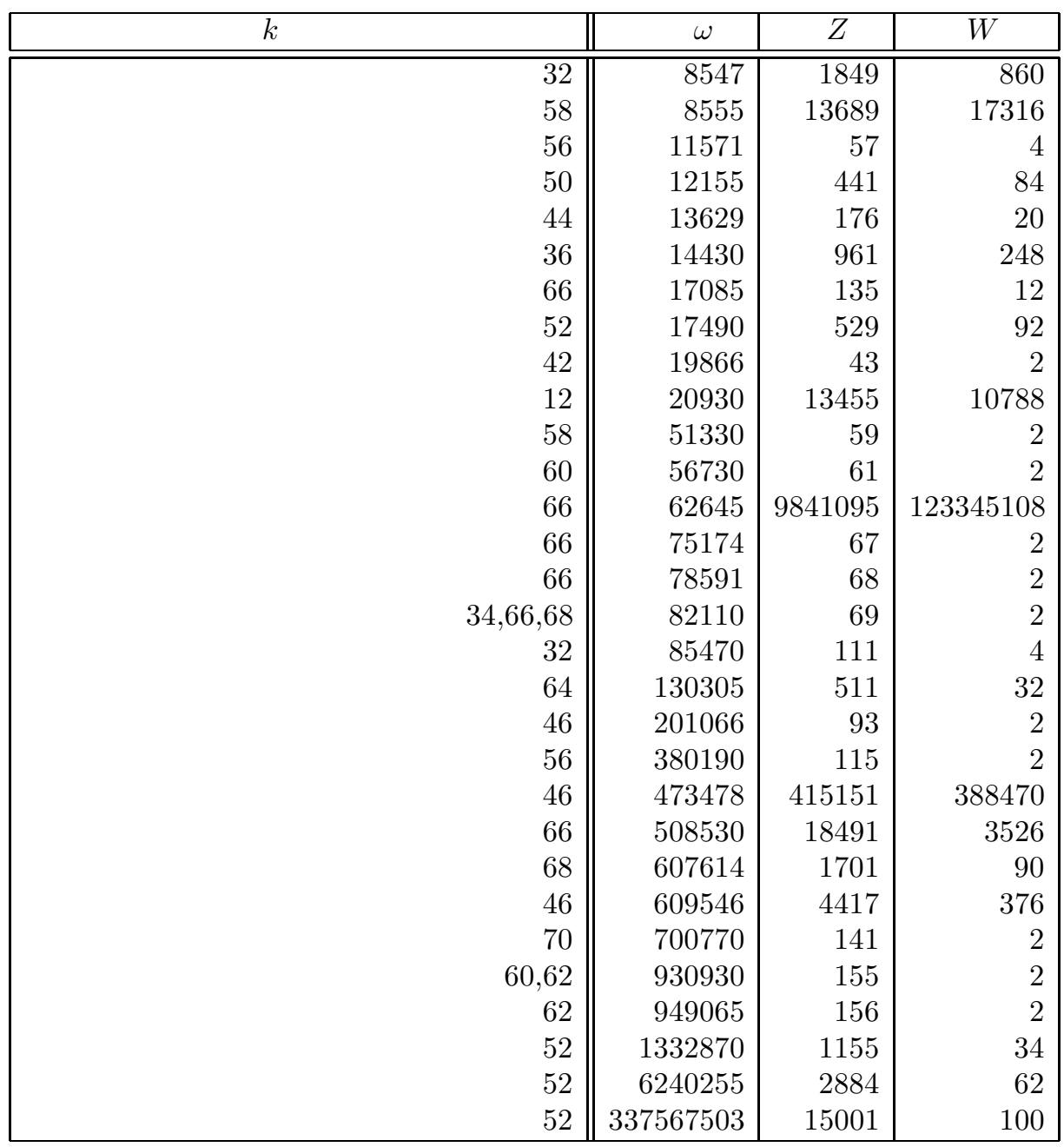

\section{REFERENCES}

1. M. A. Bennett and P. G. Walsh, The Diophantine equation $b^{2} X^{4}-d Y^{2}=1$, Proc. A.M.S., 127 (1999), 3481-3491. MR 2000b:11025

2. B. Brindza, On some generalizations of the diophantine equation $1^{k}+2^{k}+\cdots+x^{k}=y^{z}$, Acta Arith. 44 (1984), 99-107. MR 86j:11029

3. B. Brindza, Power values of the sum $1^{k}+2^{k}+\cdots+x^{k}$, in "Number Theory" (Budapest 1987), Colloq. Math. Soc. János Bolyai, vol. 51, North-Holland, Amsterdam, 1990, 595-603. MR 91g:11027

4. B. Brindza and Á. Pintér, On the number of solutions of the equation $1^{k}+2^{k}+\cdots+(x-1)^{k}=$ $y^{z}$, Publ. Math. Debrecen 56/3-4 (2000), 271-277. MR 2001i:11032

5. J. Buchmann, C. Thiel, and H. C. Williams, Short representation of quadratic integers, in "Computational Algebra and Number Theory, Mathematics and its Applications" 325, Kluwer, Dordrecht, 1995, 159-185. MR 96c:11144

6. J. H. E. Cohn, The Diophantine equation $x^{4}-D y^{2}=1$ II. Acta Arith. 78 (1997), 401-403. MR 98e: 11033

7. K. Dilcher, On a Diophantine equation involving quadratic characters, Compositio Math. $\mathbf{5 7}$ (1986), 383-403. MR 87e:11046 
8. K. Dilcher, Zeros of Bernouli, generalized Bernouli, and Euler polynomials, Mem. A.M.S., 73 no. 386 (1988) (94 pages). MR 89h:30005

9. J. Gebel, A. Pethö, and H. Zimmer, Computing integral points on elliptic curves, Acta Arith. 68 (1994), 171-192. MR 95i:11020

10. M. Voorhoeve, K. Györy and R. Tijdeman, On the diophantine equation $1^{k}+2^{k}+\cdots+x^{k}+$ $R(x)=y^{z}$, Acta Math. 143 (1979), 1-8; Corr. 159 (1987), 151-152. MR 80e:10020 \& MR 88i: 11018

11. M. J. Jacobson, Jr., Subexponential Class Group Computation in Quadratic Orders, Ph.D. thesis, Technische Universität Darmstadt, Darmstadt, Germany, 1999.

12. M. J. Jacobson, Jr., R. F. Lukes, and H. C. Williams, An investigation of bounds for the regulator of quadratic fields, Experiment. Math. 4 (1995), no. 3, 211-225. MR 97d:11173

13. M. J. Jacobson, Jr. and H. C. Williams, The size of the fundamental solutions of consecutive Pell equations, Experiment. Math. 9 (2000), no. 4, 631-640. MR 2002f:11142

14. M. J. Jacobson, Jr. and H. C. Williams, Modular arithmetic on elements of small norm in quadratic fields, Submitted to Designs, Codes, and Cryptography.

15. D. H. Lehmer, An extended theory of Lucas functions, Ann. Math. 31 (1930), 419-448.

16. H. W. Lenstra, Jr., On the calculation of regulators and class numbers of quadratic fields, London Math. Soc. Lecture Note Series 56 (1982), 123-150. MR 86g:11080

17. The LiDIA Group, LiDia: a $C++$ library for computational number theory, Software, Technische Univesität Darmstadt, Germany, 1997, see http://www.informatik.tudarmstadt.de/TI/LiDIA.

18. É. Lucas, Solution de la question 1180, Nouv. Ann. Math. (2) 16 (1877), 429-432.

19. Á. Pintér, On a conjecture of Schaffer concerning the power values of power sums, preprint, (2000).

20. J. J. Schäffer, The equation $1^{p}+2^{p}+3^{p}+\cdots+n^{p}=m^{q}$, Acta Math. 95 (1956), 155-189. MR 17:1187a

21. T. N. Shorey and R. Tijdeman, Exponential Diophantine Equations, Cambridge University Press, 87, New York, 1986. MR 88h:11002

22. J. Urbanowicz, On the equation $f(1) 1^{k}+f(2) 2^{k}+\cdots+f(x) x^{k}+R(x)=b y^{z}$, Acta Arith. 51 (1988), 349-368. MR 90b:11025

23. H. C. Williams, Édouard Lucas and Primality Testing, Canadian Mathematical Society Series of Monographs and Advanced Texts, vol. 22, John Wiley \& Sons, New York, 1998. MR 2000b:11139

Department of Computer Science, University of Calgary, 2500 University Drive NW, Calgary, Alberta, T2N 1N4 Canada

E-mail address: jacobs@cpsc.ucalgary.ca

Institute for Mathematics, University of Debrecen, P.O. Box 12, H-4010 Debrecen, HUNGARY

E-mail address: pinterak@freemail.hu

Department of Mathematics, University of Ottawa, 585 King Edward St., Ottawa, Ontario, K1N 6N5 CANADA

E-mail address: gwalsh@mathstat.uottawa.ca 